This information is current as of April 26, 2023.

\title{
Brain Stem Diffusion-Weighted Imaging Lesion Score: A Potential Marker of Outcome in Acute Basilar Artery Occlusion
}

T.-H. Cho, N. Nighoghossian, F. Tahon, C. Némoz, M. Hermier, F. Salkine, L. Derex, P. Trouillas, J.-C. Froment and F. Turjman

AJNR Am J Neuroradiol 2009, 30 (1) 194-198 doi: https://doi.org/10.3174/ajnr.A1278

http://www.ajnr.org/content/30/1/194 


\section{ORIGINAL RESEARCH}

T.-H. Cho

N. Nighoghossian

F. Tahon

C. Némoz

M. Hermier

F. Salkine

L. Derex

P. Trouillas

J.-C. Froment

F. Turjman

\section{Brain Stem Diffusion-Weighted Imaging Lesion Score: A Potential Marker of Outcome in Acute Basilar Artery Occlusion}

\begin{abstract}
BACKGROUND AND PURPOSE: The benefit of recanalization in basilar artery occlusion (BAO) has been established. The baseline extent of brain stem damage may also influence the outcome. We investigated whether a baseline diffusion-weighted imaging (DWI) score may provide additional prognostic value in $\mathrm{BAO}$

MATERIALS AND METHODS: We analyzed baseline clinical and DWI parameters in consecutive patients treated with endovascular procedures for acute BAO. Brain stem DWI lesions were assessed by using a semiquantitative score based on arterial territory segmentation. Outcome at 3 months was dichotomized according to the modified Rankin Scale (mRS) as favorable (mRS, 0-2) or unfavorable (mRS, 3-6). Spearman rank correlation tests assessed the correlation between DWI and clinical variables. Univariate and multivariate logistic regression analyses were used to identify clinical and MR imaging predictors of outcome.
\end{abstract}

RESULTS: Twenty-nine patients were included. The brain stem DWI score (median, 3; range, 0-14) was correlated with the baseline National Institutes of Health Stroke Scale (NIHSS) score and the presence and length of coma ( $r=0.67,0.49$, and 0.53 , respectively; $P<.01)$. Recanalization was achieved in $76 \%$. A higher baseline NIHSS score $(P=.02)$ and brain stem DWI score $(P=.03)$, a lower Glasgow Coma Scale score $(P=.04)$, and the presence of coma $(P=.05)$ were associated with poor outcome in univariate analysis. Multivariate analysis showed that the brain stem DWI score was the only independent baseline predictor for clinical outcome $(P=.026)$.

CONCLUSIONS: Baseline brain stem DWI lesion score is an independent marker of outcome in BAO
A cute basilar artery occlusion (BAO) is a devastating disease, ${ }^{1}$ with mortality reaching $80 \%-90 \%$ in patients without recanalization. ${ }^{2,3}$ Acute $\mathrm{BAO}$ accounts for $6 \%-10 \%$ of stroke in trials of intracranial large-vessel occlusion. ${ }^{4,5}$ Intraarterial thrombolysis (IAT) and percutaneous transluminal angioplasty/stent placement have decreased BAO mortality to $30 \%-65 \%$. $^{6-12}$ Fewer data are available for intravenous thrombolysis (IVT), ${ }^{13-15}$ but a recent meta-analysis showed no significant difference in outcome between IVT and IAT, despite higher recanalization rates with IAT. ${ }^{11}$ To our knowledge, no randomized study comparing IVT and IAT is available.

Early recanalization is the best documented predictor of good outcome in BAO. Baseline ischemic damage may also influence the prognosis because an extensive brain stem lesion may diminish the benefits of recanalization. The prognostic value of DWI and perfusion-weighted imaging (PWI) has been demonstrated in patients with acute anterior ischemic stroke. Patients with an adequate DWI-PWI mismatch benefited from reperfusion, whereas those without a PWI-DWI mismatch showed no benefit from therapy. ${ }^{16}$ Moreover, patients with a large PWI and/or DWI lesion, with or without a

Received May 23, 2008; accepted after revision July 15.

From the Cerebrovascular Unit (T.-H.C., N.N., L.D., P.T.) and Department of Radiology (F.Tahon, M.H., F.S., J.-C.F., F.Turjman), Hôpital Neurologique Pierre Wertheimer, Lyon, France; CREATIS (T.-H.C., N.N., M.H., L.D., J.-C.F.), UMR 5515, INSERM U 630, Université Claude Bernard, Lyon, France; and Laboratoire de Biostatistiques de la Santé (C.N.), UMR CNRS 5558, Lyon, France.

Please address correspondence to Norbert Nighoghossian, MD, PhD, Hôpital Neurologique Pierre Wertheimer, 59 Bd Pinel, 69003 Lyon, France; e-mail: norbert.nighoghossian@ chu-lyon.fr

Indicates article with on-line table.

DOI 10.3174/ajnr.A1278
PWI-DWI mismatch, appeared at higher risk of intracranial hemorrhage after IVT. ${ }^{16}$ Multitechnique MR imaging has also been used in posterior circulation stroke, ${ }^{17-20}$ but no prospective series has validated its predictive value. Acute global DWI lesion volume did not correlate with the baseline National Institutes of Health Stroke Scale (NIHSS) score in previous studies of vertebrobasilar ischemic stroke. ${ }^{19,21}$ A score based on arterial territory segmentation may be more appropriate. We assessed the prognostic impact of baseline brain stem DWI scores in consecutive patients treated with endovascular therapy for acute BAO.

\section{Materials and Methods}

\section{Patients}

From November 2003 to June 2007, consecutive patients who underwent MR imaging before treatment with endovascular procedures for acute $\mathrm{BAO}$ were included in the present study. Inclusion criteria were the following: 1) brain stem or cerebellar symptoms with onset within 48 hours before treatment; 2) MR imaging including DWI and MR angiography completed before treatment; and 3) BAO confirmed by intra-arterial digital substraction angiography (DSA) and treated by endovascular approaches. Exclusion criteria included the following: 1) evidence of any intracranial hemorrhage on pretreatment MR imaging, and 2) clinical or laboratory contraindication for IAT. Informed consent was obtained from the patient or relatives before treatment. During the study period, 37 consecutive patients underwent endovascular therapy for vertebrobasilar stroke. Among these, 2 patients had a P1 occlusion without BAO and were excluded. Among the remaining 35 patients, 6 had a CT scan instead of MR imaging (agitated or hemodynamically unstable, $n=4$; unavailable MR imaging, $n=2$ ). Twenty-nine patients were eventually included. 

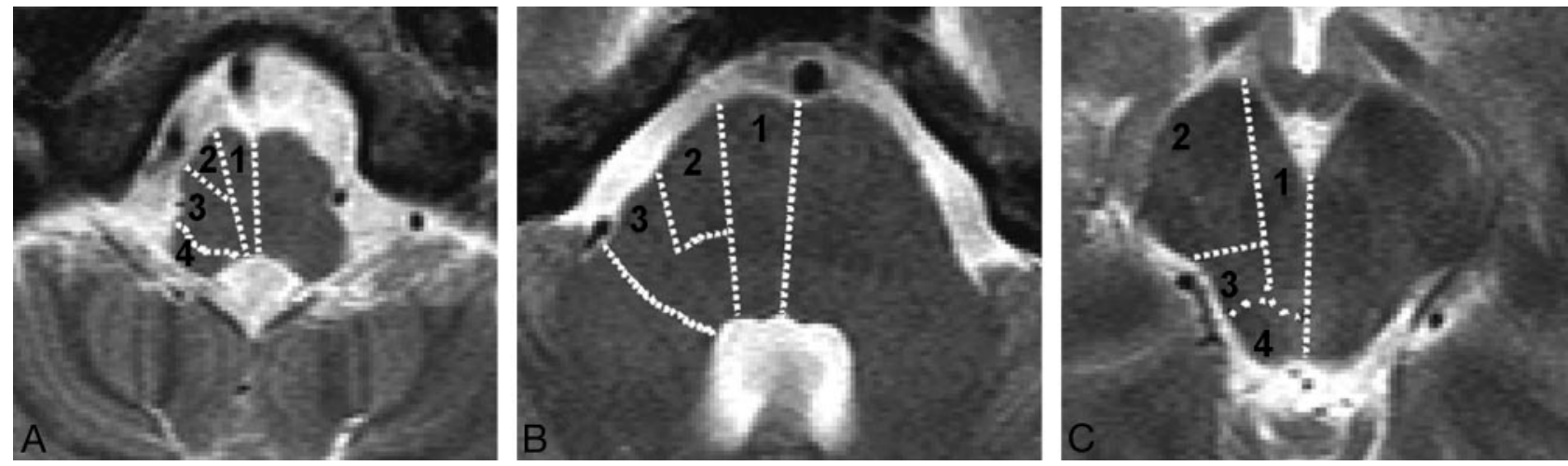

Fig 1. Brain stem DWI lesion score. $A$, Medulla. B, Pons. C, Midbrain. Arterial segmentation according to Tatu et al ${ }^{22}$ : 1 indicates anteromedial; 2 , anterolateral; 3 , lateral; and 4 , posterior territories. The total number of brain stem arterial territories with abnormal DWI defines the brain stem DWI lesion score, between 0 and 22.

Baseline NIHSS score and Glasgow Coma Scale (GCS) were assessed by a trained stroke neurologist on admission. Age, pretreatment duration of coma, and time from symptom onset to treatment were recorded. Clinical outcome was assessed by the modified Rankin Scale (mRS) at 3 months and dichotomized as favorable (mRS, 0-2) or unfavorable (mRS, 3-6). Symptomatic intracranial hemorrhage $(\mathrm{SIH})$ was defined as a neurologic deterioration $(\geq 4$ points in the NIHSS) associated with intracranial bleeding on control CT.

\section{Imaging Protocol}

MR imaging examination used an Intera 1.5T imager (Philips Medical Systems, Best, the Netherlands). The following sequences were performed:

1) DWI sequence: 3 -directional single-shot echo-planar: TR, 3082 $\mathrm{ms}$; TE, $74 \mathrm{~ms}$; flip angle, $90^{\circ} ; 3$ values of $\mathrm{b}\left(0,500\right.$ and $\left.1000 \mathrm{~s} / \mathrm{mm}^{2}\right)$ used to calculate the apparent diffusion coefficient (ADC) maps; echo planar imaging factor, 69; FOV, $230 \mathrm{~mm}$; matrix, 256; 22 sections; section thickness, $5 \mathrm{~mm}$; imaging time, 1 minute 44 seconds.

2) $\mathrm{T} 2{ }^{\star}$-weighted sequence: TR, $800 \mathrm{~ms}$; TE, $28 \mathrm{~ms}$; flip angle, $20^{\circ}$; FOV, $230 \mathrm{~mm}$; matrix, 512; 22 sections; section thickness, $5 \mathrm{~mm}$; imaging time, 2 minutes 11 seconds.

3) $3 \mathrm{D}$ time-of-flight angiography (without gadolinium): TR, $25 \mathrm{~ms}$; TE, $3.1 \mathrm{~ms}$; section thickness, $0.5 \mathrm{~mm}$; 140 sections; FOV, $160 \mathrm{~mm}$; scan matrix, 360; reconstruction matrix, 512; imaging time, 4 minutes 14 seconds.

Selective diagnostic 4-vessel DSA was performed in all cases. Thrombolysis in Myocardial Infarction (TIMI) grades were used to assess recanalization of the basilar artery. TIMI grades $0-1$ were classified as recanalization failure and TIMI grades $2-3$ defined successful recanalization. CT was performed on days 1 and 7 to rule out bleeding.

\section{Image Analysis: DWI Lesion Scoring}

A semiquantitative scale was used to evaluate the extent of the brain stem DWI lesion on pretreatment MR imaging according to the arterial territories of the medulla, pons, and midbrain as described by Tatu et al (Fig 1). ${ }^{22}$ The number of arterial territories with abnormal DWI was assessed at each brain stem level: $0-8$ in the medulla, $0-6$ in the pons, and $0-8$ in the midbrain. The total number of involved arterial territories defined the brain stem DWI lesion score, comprising scores between 0 and 22. Cerebellar DWI lesions were dichotomized as minor (less than one third of a cerebellar hemisphere) or major (more than one third of a cerebellar hemisphere). MR imaging analysis was done by 2 experienced observers blinded to the severity of the clinical deficit. The interobserver and intraobserver variations were assessed by comparing the differences in measurement 10 days apart on the whole sample. For the discrepant cases, the final scoring of DWI data was achieved by a consensual analysis.

\section{Therapeutic Regimen}

Treatment of acute BAO involved several methods with time, reflecting the development of new strategies and the degree of early response to the initial recanalization procedures. We used the following intraarterial treatment regimens: 1) tissue plasminogen activator (tPA) alone ( $n=5$; mean dosage, $34 \pm 11 \mathrm{mg}$ ), 2) tPA with intravenous abciximab $(n=4$; mean tPA dosage, $30 \pm 12 \mathrm{mg}$; mean abciximab dosage, $23 \pm 6 \mathrm{mg}$ ), 3) tPA with a mechanical procedure (angioplasty, thromboaspiration, snare microcatheter, $n=16$; mean tPA dosage, $30 \pm 16 \mathrm{mg})$, or 4$)$ mechanical procedure only $(n=4)$. Patients were transferred to the neurologic intensive care unit following the procedure. Prophylactic anticoagulation was started 24 hours after IAT and consisted of enoxaparin (4000 IU/day) and acetylsalicylic acid (100$300 \mathrm{mg}$ /day) or clopidogrel (75 mg/day). Unfractionated heparin was administered in patients with documented atrial fibrillation.

\section{Statistical Analysis}

Descriptive statistics of clinical and DWI parameters are given as median values with ranges, unless indicated otherwise. Spearman rank correlation tests assessed the correlation between DWI parameters and clinical variables. The relationship between $\mathrm{SIH}$ and baseline variables (DWI data, NIHSS score, time to treatment) was assessed with Wilcoxon rank sum and Fisher exact tests. Univariate and multivariate logistic regression analyses were used to identify DWI and clinical parameters most closely associated with the final mRS score. The following explanatory variables were systematically assessed by univariate logistic regression analysis: age, NIHSS score, GCS score, presence or absence of coma (GCS score, $<8$ ), duration of coma before treatment, time to treatment, brain stem DWI lesion score, presence or absence of minor/major cerebellar lesions, and recanalization. Relevant univariate factors $(P<.05)$ were included in the multivariate analysis, by using a backward stepwise method. Statistical analyses were performed with the Statistical Package for the Social Sciences (Version 11.5 for Windows; SPSS, Chicago, Ill). 

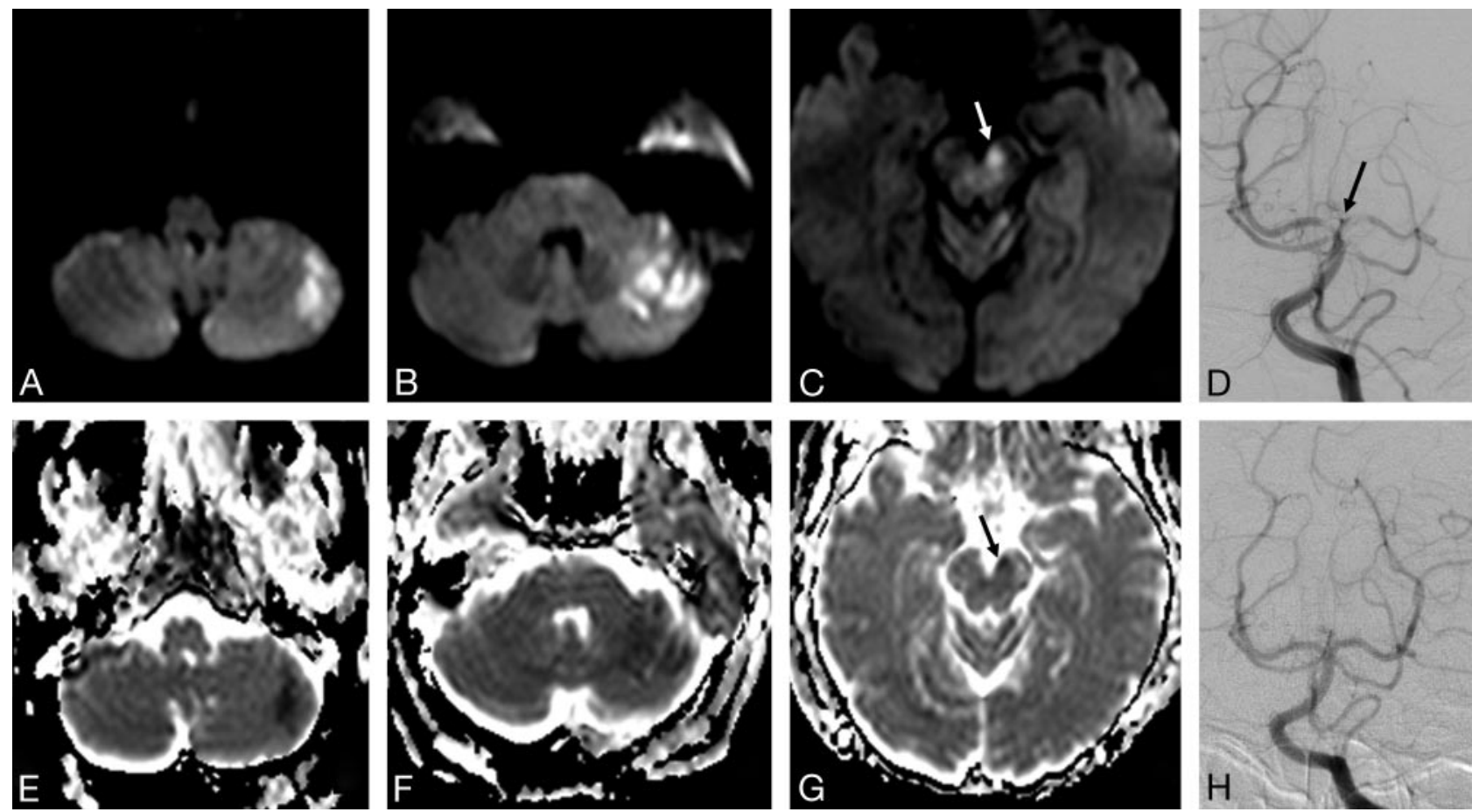

Fig 2. Pretreatment DWI $(A-C)$ and $A D C$ maps $(E-G)$ of a 70-year-old woman with right hemiparesis, dysarthria, diplopia, and reduced consciousness. DWI shows limited lesions in the midbrain (arrows, $C$ and $G$; brain stem DWI lesion score, 1) and in the left cerebellar hemisphere. DSA shows a distal basilar clot (arrow, D) successfully recanalized after intra-arterial tPA (H). Time from symptom onset to treatment was 13 hours 30 minutes. Outcome was favorable at 3 months (mRS, 2).

\section{Results}

\section{Baseline Clinical and DWI Data}

Twenty-nine patients with acute BAO (13 women, 16 men) met study criteria. Their median age was 60 years (range, 26-77 years). Median NIHSS score was 22 (range, 4-34). Coma was present in $13 / 29(45 \%)$ patients, with a median duration of coma before treatment of 6.4 hours (range, 2-18 hours). Median brain stem DWI score was 3 (range, 0-14). The intraobserver and interobserver reliabilities in measured brain stem DWI lesion scores were $r>0.9$. Major cerebellar DWI lesions were observed in 12/29 (41.4\%). Clinical, vascular, DWI data, and outcome are summarized in the on-line Table. Illustrative cases are given in Figs 2 and 3.

\section{Recanalization Rate, Clinical Outcome, and Bleeding Complications}

The sites of basilar occlusion were as follows: lower basilar, $n=18$; midbasilar, $n=5$; upper basilar arteries, $n=6$. Median time to treatment was 7.5 hours (range, 3.2-26.6 hours). There was no significant difference in the mean time to treatment between patients with favorable versus unfavorable outcomes: $7.6 \pm 3.9$ hours versus $9.4 \pm 5.5$ hours $(P=.41)$. Recanalization was achieved in 22/29 (76\%) patients. Lower basilar occlusion was recanalized significantly less often $(P=$ .018). Mean time to treatment showed no significant difference among patients with successful or failed recanalization ( $9 \pm 5.5$ hours versus $8.5 \pm 4.3$ hours, respectively, $P=.959$ ). A favorable outcome ( $\mathrm{mRS}, 0-2$ ) was observed in $8 / 22$ recanalized patients versus $0 / 7$ patients ( 2 patients were locked in; 5 died) without recanalization $(P=.142)$. Overall mortality was $14 / 29$ patients $(48 \%)$. Death related to SIH occurred in 5 patients. SIH showed no significant association with the brain stem DWI score ( $P=.449$, Wilcoxon rank sum test), cerebellar damage $(P=.622$, Fisher exact test), NIHSS score $(P=$ .298 , Wilcoxon rank sum test), or time to treatment $(P=.204$, Wilcoxon rank sum test). No systemic hemorrhagic complication was observed.

\section{Correlation between DWI Scoring and Clinical Variables}

The brain stem DWI lesion score was correlated with the baseline NIHSS score and with the presence and length of coma before treatment $(r=0.67,0.49$, and 0.53 , respectively; $P<$ $.01)$. No significant correlation was found between the brain stem DWI lesion score and the recanalization rate or time to treatment.

\section{Baseline Predictors}

Univariate analysis showed that a higher baseline NIHSS score $(P=.02)$, a higher brain stem DWI lesion score $(P=.03)$, a lower GCS score $(P=.04)$, and the presence of coma $(P=.05)$ were significantly associated with an unfavorable outcome (Table 1). Conversely, age, length of coma, time to treatment, cerebellar damage, and recanalization did not correlate with clinical outcome in univariate regression analysis. Significant variables in univariate analyses were included in the multivariate model (NIHSS score, brain stem DWI score, GCS score, and coma on admission). The multivariate analysis showed that the brain stem DWI lesion score was the only independent baseline predictor for clinical outcome (Table 2, $P=.026$ ).

\section{Discussion}

The present study reports the largest baseline DWI data available in acute BAO. The extent of the brain stem DWI lesion was assessed by using a simple and reliable scoring system 

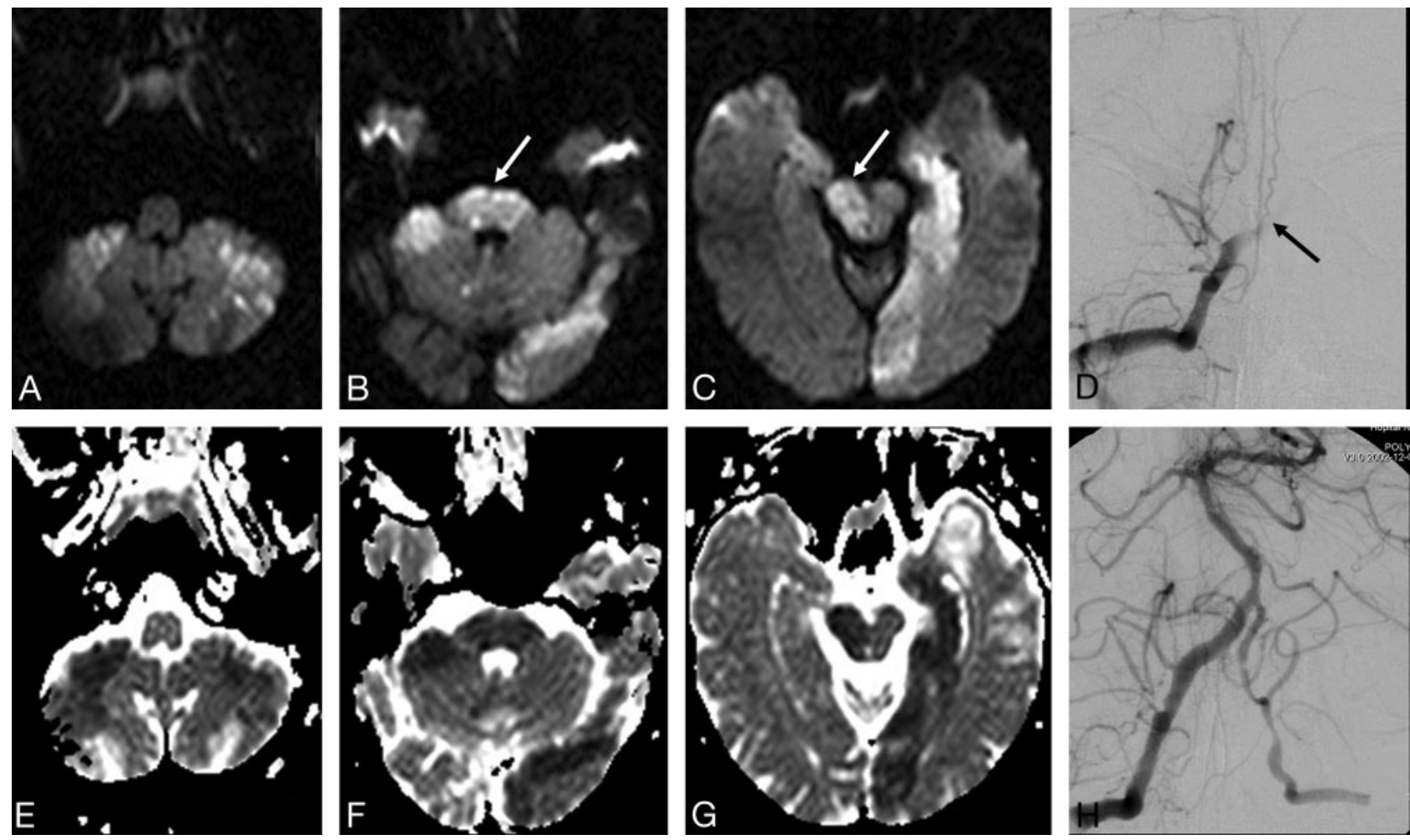

Fig 3. Pretreatment DWI $(A-C)$ and $A D C$ maps $(E-G)$ of a comatose 45 -year-old man with areactive tetraplegia. DWI shows extensive brain stem lesions (arrows, $B$ and $C$; brain stem DWI lesion score, 10) and bilateral lesions in the posterior inferior cerebellar arteries and left posterior cerebral artery territories. DSA shows occlusion of the V4 segment of the right vertebral artery and of the lower basilar artery (arrow, D). A TIMI 2 recanalization was achieved after intra-arterial tPA and balloon angioplasty of a 70\% proximal basilar stenosis ( $\mathrm{H})$. Time from symptom onset to treatment was 14 hours 20 minutes. The patient was in a locked-in syndrome at 3 months (mRS, 5).

\begin{tabular}{lcc}
\multicolumn{2}{l}{ Table 1: Univariate regression analysis } \\
\hline $\begin{array}{l}\text { Prognostic } \\
\text { Variables }\end{array}$ & $\begin{array}{c}\text { Odds Ratio } \\
(95 \% \mathrm{CI})\end{array}$ & $P$ Value \\
\hline NIHSS score & $1.12(1.02-1.24)$ & .02 \\
Brain stem DWI score & $1.94(1.08-3.48)$ & .03 \\
GCS & $0.80(0.64-0.99)$ & .04 \\
Presence of coma & $9.33(0.97-90.0)$ & .05 \\
\hline
\end{tabular}

Note:-Cl indicates confidence interval; GCS, Glasgow Coma Scale Score.

\begin{tabular}{lcc}
\hline \multicolumn{2}{l}{ Table 2: Multivariate regression analysis } \\
\hline Variable & $P$ Value & $\begin{array}{c}\text { Odds Ratio } \\
(95 \% \mathrm{Cl})\end{array}$ \\
\hline Brain stem DWI score & .026 & $1.942(1.084-3.481)$
\end{tabular}

without additional time-consuming postprocessing. Multivariate regression analysis showed that the extent of the pretreatment brain stem DWI lesion was the strongest baseline predictor of outcome. A study of 10 patients with BAO failed to show a significant relationship between baseline DWI lesion volume and NIHSS score. ${ }^{19}$ In contrast, we found a significant association between the brain stem DWI lesion score and the NIHSS score. This discrepancy might be explained by a scoring system based on arterial segmentation rather than a global volume assessment. Indeed, clinical severity and outcome in $\mathrm{BAO}$ are likely influenced by strategic brain stem damage (Figs 2 and 3). The correlation between brain stem DWI score and coma duration may also support this view. A recent study by Renard $\mathrm{et}^{2{ }^{23}}$ used a 10-point semiquantitative scoring of baseline DWI in 16 patients with acute BAO: 1 point was given for each half of the medulla, pons, and midbrain. Their score included thalamic and temporo-occipital lesions ( 0.5 point for each structure) as well as cerebellar damage ( 1 point for unilateral and 2 for bilateral lesions). A total score of $>3$ was associated with poor outcome ( $\mathrm{mRS}, \geq 3$ ) in univariate analysis. The sample size precluded a multivariate analysis. Both studies support the use of a sectorial scoring of DWI lesions in $\mathrm{BAO}$ rather than a volume measurement. A score based on arterial territory segmentation was preferred in the present study. We showed, in addition, that baseline brain stem damage was an independent predictor of outcome.

Formerly described clinical predictors were confirmed: Higher baseline NIHSS score and presence of coma were predictive of poor outcome. ${ }^{7,10}$ Recanalization was achieved in $22 / 29(76 \%)$ patients, in line with previous studies using interventional approaches. ${ }^{7-10,12}$ Although no significant association between recanalization and outcome was observed in our series, probably due to the small sample size, no patient with failed recanalization achieved functional independence.

Time to treatment was correlated with neither the brain stem DWI score nor outcome. Our sample size and the proportion of "progressive" strokes in our series (41\%) may explain these results. The occlusion level and degree of collateral circulation likely account for the wide spectrum of clinical presentations in BAO. The prognosis of patients with early severe symptoms may be more time-dependent than those with a more protracted evolution.

Our results may have significant implications in acute BAO management. Treatment strategies show great variations in different hospitals regarding the method (intra-arterial versus intravenous procedures) and time window for eligibility. Al- 
though recanalization may favor partial reversibility of the DWI anomaly, ${ }^{24}$ the baseline brain stem DWI score may help to identify patients most likely to benefit from treatment. Extensive brain stem damage may indicate poor prognosis regardless of recanalization success or failure (Fig 3). Conversely, patients with limited DWI lesions might benefit from late reperfusion therapy (Fig 2). Nevertheless, clinical implementation of the brain stem DWI score will require further validation with prospective studies. DWI data need to be interpreted together with clinical parameters. Treatment should not be denied on the sole basis of MR imaging results. Accordingly, in this consecutive patient series, no one was denied a recanalization attempt after MR imaging. Patients with limited or extensive brain stem damage were treated both early and late after symptom onset.

\section{Study Limitations}

As in most studies of acute BAO, the sample size is the main limitation. More prospective data are needed to delineate the prognostic role of acute DWI in BAO. Lack of uniformity in the treatment regimen may have affected the correlation between the DWI lesion score and outcome, because varying therapies could yield different recanalization rates and outcomes. The different treatment-regimen efficacy could not be assessed in our study due to the limited sample size. Nevertheless, because recanalization and brain stem DWI scores showed no significant correlation, it seems unlikely that the treatment regimen influenced the correlation between DWI score and outcome.

\section{Conclusions}

We showed that the brain stem DWI score is an independent baseline predictor of outcome in acute $\mathrm{BAO}$, without correlation to recanalization. In light of previous data, the present study suggests that both pretreatment brain stem damage and reperfusion are major independent determinants of clinical outcome. A DWI score based on arterial territory segmentation appears more appropriate than volume measurements in BAO. Further prospective validation is needed before its use in treatment decisions.

\section{Acknowledgments}

We thank Michèle Canova for her help in collecting and processing the data and Mark Inglin for editing assistance.

\section{References}

1. Kubik CS, Adams RD. Occlusion of the basilar artery: a clinical and pathological study. Brain 1946;59:73-121

2. Zeumer H, Hacke W, Kolmann HL, et al. Local fibrinolysis in basilar artery thrombosis (author's transl) [in German]. Dtsch Med Wochenschr 1982;107: 728-31

3. Hacke W, Zeumer H, Ferbert A, et al. Intra-arterial thrombolytic therapy improves outcome in patients with acute vertebrobasilar occlusive disease. Stroke 1988;19:1216-22

4. IMS Study Investigators. Combined intravenous and intra-arterial recanalization for acute ischemic stroke: the Interventional Management of Stroke Study. Stroke 2004;35:904-11. Epub 2004 Mar 11

5. Flint AC, Duckwiler GR, Budzik RF, et al, for the MERCI and Multi MERCI Writing Committee. Mechanical thrombectomy of intracranial internal carotid occlusion: pooled results of the MERCI and Multi MERCI Part I trials. Stroke 2007;38:1274-80. Epub 2007 Mar 1

6. Brandt T, von Kummer R, Müller-Küppers M, et al. Thrombolytic therapy of acute basilar artery occlusion: variables affecting recanalization and outcome. Stroke 1996;27:875-81

7. Arnold M, Nedeltchev K, Schroth G, et al. Clinical and radiological predictors of recanalisation and outcome of 40 patients with acute basilar artery occlusion treated with intra-arterial thrombolysis. J Neurol Neurosurg Psychiatry 2004;75:857-62

8. Sliwka U, Mull M, Stelzer A, et al. Long-term follow-up of patients after intraarterial thrombolytic therapy of acute vertebrobasilar artery occlusion. Cerebrovasc Dis 2001;12:214-19

9. Eckert B, Koch C, Thomalla G, et al. Aggressive therapy with intravenous abciximab and intra-arterial rtPA and additional PTA/stenting improves clinical outcome in acute vertebrobasilar occlusion: combined local fibrinolysis and intravenous abciximab in acute vertebrobasilar stroke treatment (FAST)—results of a multicenter study. Stroke 2005;36:1160-65. Epub 2005 May 12

10. Schulte-Altedorneburg G, Hamann GF, Mull M, et al. Outcome of acute vertebrobasilar occlusions treated with intra-arterial fibrinolysis in 180 patients. AJNR Am J Neuroradiol 2006;27:2042-47

11. Lindsberg PJ, Mattle HP. Therapy of basilar artery occlusion: a systematic analysis comparing intra-arterial and intravenous thrombolysis. Stroke 2006;37:922-28

12. Müller R, Pfefferkorn T, Vatankhah B, et al. Admission facility is associated with outcome of basilar artery occlusion. Stroke 2007;38:1380-83

13. Grond M, Rudolf J, Schmülling S, et al. Early intravenous thrombolysis with recombinant tissue-type plasminogen activator in vertebrobasilar ischemic stroke. Arch Neurol 1998;55:466-69

14. Montavont A, Nighoghossian N, Derex L, et al. Intravenous r-TPA in vertebrobasilar acute infarcts. Neurology 2004;62:1854-56

15. Lindsberg PJ, Soinne L, Tatlisumak T, et al. Long-term outcome after intravenous thrombolysis of basilar artery occlusion. JAMA 2004;292:1862-66

16. Albers GW, Thijs VN, Wechsler L, et al, for the DEFUSE Investigators. Magnetic resonance imaging profiles predict clinical response to early reperfusion: the diffusion and perfusion imaging evaluation for understanding stroke evolution (DEFUSE) study. Ann Neurol 2006;60:508-17

17. Nighoghossian N, Derex L, Turjman F, et al. Hyperacute diffusion-weighted MRI in basilar occlusion treated with intra-arterial t-PA. Cerebrovasc Dis 1999;9:351-54

18. Du Mesnil de Rochemont R, Neumann-Haefelin T, Berkefeld J, et al. Magnetic resonance imaging in basilar artery occlusion. Arch Neurol 2002;59:398-402

19. Ostrem JL, Saver JL, Alger JR, et al. Acute basilar artery occlusion: diffusionperfusion MRI characterization of tissue salvage in patients receiving intraarterial stroke therapies. Stroke 2004;35:30-34

20. Suzuki S, Kidwell CS, Starkman S, et al. Use of multimodal MRI and novel endovascular therapies in a patient ineligible for intravenous tissue plasminogen activator. Stroke 2005;36:e77-79

21. Linfante I, Llinas RH, Schlaug G, et al. Diffusion-weighted imaging and National Institutes of Health Stroke Scale in the acute phase of posteriorcirculation stroke. Arch Neurol 2001;58:621-28

22. Tatu L, Moulin T, Bogousslavsky J, et al. Arterial territories of human brain: brainstem and cerebellum. Neurology 1996;47:1125-35

23. Renard D, Landragin N, Robinson A, et al. MRI-based score for acute basilar artery thrombosis. Cerebrovasc Dis 2008;25:511-16

24. Pialat JB, Wiart M, Nighoghossian N, et al. Evolution of lesion volume in acute stroke treated by intravenous t-PA. J Magn Reson Imaging 2005;22:23-28 